\title{
CHARACTERIZATIONS OF QUASI-METRIZABLE BITOPOLOGICAL SPACES
}

\author{
T. G. RAGHAVAN and I. L. REILLY
}

(Received 15 September 1986)

Communicated by J. H. Rubinstein

\begin{abstract}
In this paper we prove that a pairwise Hausdorff bitopological space $\left(X, \mathscr{T}_{1}, \mathscr{T}_{2}\right)$ is quasi-metrizable if and only if for each point $x \in X$ and for $i, j=1,2, i \neq j$, one can assign $\mathscr{T}_{i}$ nbd bases $\{S(n, i ; x) \mid n=1,2, \ldots\}$ such that (i) $y \notin S(n-1, i ; x)$ implies $S(n, i ; x) \cap S(n, j ; y)=\phi$, (ii) $y \in S(n, i ; x)$ implies $S(n, i ; y) \subset S(n-1, i ; x)$. We derive two further results from this.

1980 Mathematics subject classification (Amer. Math. Soc.): 54 E 55.

Keywords and phrases: quasi-metric, quasi-uniformity, bitopological space.

The concept of quasi-metric spaces was first introduced by Wilson [11]. The fact that a quasi-metric gives rise to a conjugate quasi-metric was noticed by Kelly [1], thus leading to the study of bitopological spaces. Since then one of the main problems in this area has been to find necessary and sufficient conditions for quasi-metrization. This problem was considered by Kelly [1] Patty [5], Lane [2], Reilly [6], Salbany [9] and later by Pareek [4] and Romaguera [7, 8].

The related notion of quasi-uniform spaces and their properties have been discussed in great detail in Murdheswar and Naimpally [3] and Stoltenberg [10]. In the proof of Theorem 1 we make use of the quasi-uniform analogue of the metrization theorem of Alexandroff and Urysohn, namely, a pairwise Hausdorff quasi-uniform space $\left(X, \mathscr{V}, \mathscr{V}^{-1}\right)$ is quasi-metrizable if and only if $\mathscr{V}$ has a countable base. From Theorem 1 we derive Theorems 2 and 3 as corollaries. It must be noted that Theorem 2 has been proved by Pareek [4].

We write nbd for neighbourhood. If $A$ is a subset of $X$ and $\mathscr{T}_{i}$ is a topology on $X$, then $\mathscr{T}_{i} \operatorname{cl} A\left(\mathscr{T}_{i}\right.$ int $\left.A\right)$ is the closure (interior) of $A$ in the space $\left(X, \mathscr{T}_{i}\right)$.
\end{abstract}

(C) 1988 Australian Mathematical Society $0263-6115 / 88 \$ A 2.00+0.00$ 
The letters $m, n, n_{i}, m_{j}, n_{j}$ represent positive integers. The letters $i, j$ always take the values $i, j=1,2 ; i \neq j . S(n, i ; x)$ represents a $\mathscr{T}_{i}$ nbd of $x$ where $n$ is a positive integer.

1. TheOREM. A pairwise Hausdorff bitopological space $\left(X, \mathscr{T}_{1}, \mathscr{T}_{2}\right)$ is quasimetrizable if and only if for each point $x \in X$ one can assign $\mathscr{T}_{i}$ neighbourhood bases $\{S(n, i ; x) \mid n=1,2, \ldots\}$ such that

(i) $y \notin S(n-1, i ; x)$ implies $S(n, i ; x) \cap S(n, j ; y)=\varnothing$,

(ii) $y \in S(n, i ; x)$ implies $S(n, i ; y) \subset S(n-1, i ; x)(i, j=1,2 ; i \neq j)$.

Proof. To prove that the conditions are sufficient, we show first that $\left(X, \mathscr{T}_{1}, \mathscr{T}_{2}\right)$ is pairwise regular. If $S(n, i ; x) \cap S(n, j ; y)=\varnothing$, then $S(n, i ; x)$ $\subset X-S(n, j ; y)$ so that $\mathscr{T}_{j} \operatorname{cl} S(n, i ; X) \subset X-\mathscr{T}_{j}$ int $S(n, j ; y)$. Thus if $y \notin S(n-1, i ; x)$, then $y \notin \mathscr{T}_{j} \operatorname{cl} S(n, i ; x)$ so that

$$
x \in S(n, i ; x) \subset \mathscr{T}_{j} \mathrm{cl} S(n, i ; x) \subset S(n-1, i ; x) .
$$

Furthermore the space is pairwise normal. Indeed, if $A$ and $B$ are $\mathscr{T}_{1}$ closed and $\mathscr{T}_{2}$ closed subsets (of $X$ ) respectively such that $A \cap B=\varnothing$ and $y \in B$, then there exists a positive integer $n(y)$ such that $A \cap \mathscr{T}_{2} \operatorname{cl} S(n(y), 1 ; y)=\varnothing$. Since $x \notin S(n(y), 1 ; y)$ for each $x \in A, S(n(y)+1,1 ; y) \cap S(n(y)+1,2$; $x)=\varnothing$ for all $x \in A$. If $Q_{n(y)}=\cup\left\{\mathscr{T}_{2}\right.$ int $\left.S(n(y)+1,2 ; x) \mid x \in A\right\}$, then $Q_{n(y)} \supset A$ and $Q_{n(y)} \cap \mathscr{T}_{2}$ cl $S(n(y)+1,1 ; y)=\varnothing$. If we write $\cup\left\{\mathscr{T}_{1}\right.$ int $\left.S(n(y)+1,1 ; y) \mid n(y)=k\right\}=W(k, 1)$, then $Q_{k} \cap \mathscr{T}_{2} \operatorname{cl} W(k, 1)=$ $\varnothing$ so that we get a $\mathscr{T}_{1}$ open covering $\{W(k, 1) \mid k=1,2, \ldots\}$ of $B$ such that $A \cap \mathscr{T}_{2} \mathrm{cl} W(k, 1)=\varnothing$ for each $k$. Similarly we can form a $\mathscr{T}_{2}$ open covering $\{W(k, 2) \mid k=1,2 \ldots\}$ of $A$ such that $B \cap \mathscr{T}_{1} \mathrm{cl} W(k, 2)=\varnothing$ for each $k$. Then a standard argument produces disjoint sets $W_{1} \in \mathscr{T}_{1}$ and $W_{2} \in \mathscr{T}_{2}$ such that $W_{1} \supset B$ and $W_{2} \supset A$.

Let $\mathscr{K}(m, i)=\left\{\mathscr{T}_{i}\right.$ int $\left.S(m, i ; y) \mid y \in X\right\}$. Let $S(x, \mathscr{K}(m, i))=$ $\cup\left\{\mathscr{T}_{i}\right.$ int $S(m, i ; y) \mid x \in \mathscr{T}_{j}$ int $\left.S(m, j ; y)\right\}$. Let $\mathscr{B}(i ; x)=\{S(x, \mathscr{K}(m, i)) \mid m$ $=1,2 \ldots\}$. We claim $\mathscr{B}(i ; x)$ is a $\mathscr{T}_{i}$ local base at $x$. If $x$ is fixed initially and $U(i ; x)$ are arbitrary $\mathscr{T}_{i}$ nbds of $x$ then there exists $n_{i}$ such that $x \in S\left(n_{i}-1, i\right.$; $x) \subset U(i ; x)$. Consider $m=\max \left(n_{1}+1, n_{2}+1\right)$. Then clearly $S(m, i ; x) \subset$ $S\left(n_{i}, i ; x\right)$. In order to avoid confusion, let us now prove specifically $\mathscr{B}(2 ; x)$ is a $\mathscr{T}_{2}$ local base at $x$. Let $y$ be such that $x \in \mathscr{T}_{1}$ int $S(m, 1 ; y)$. Then $S(m, 1$; $y) \cap S(m, 2 ; x) \neq \varnothing$ so that $y \in S(m-1,2 ; x) \subset S\left(n_{2}, 2 ; x\right)$. Hence $S\left(n_{2}, 2\right.$; $y) \subset S\left(n_{2}-1,2 ; x\right)$. Since $m=\max \left(n_{1}+1, n_{2}+1\right), \mathscr{T}_{2}$ int $S(m, 2 ; y) \subset$ $S\left(n_{2}, 2 ; y\right) \subset S\left(n_{2}-1,2 ; x\right) \subset U(2 ; x)$. Thus $\mathscr{B}(2 ; x)$ is a $\mathscr{T}_{2}$ local base at $x$. If $x \in \mathscr{T}_{1}$ int $S(n+2,1 ; y)$, then $S(n+2,1 ; y) \cap S(n+2,2 ; x) \neq \varnothing$ so that by (i) $y \in S(n+1,2 ; x)$. Hence by (ii) $S(n+1,2 ; y) \subset S(n, 2 ; x)$ so that $\bigcup\left\{\mathscr{T}_{2}\right.$ int $S(n+2,2 ; y) \mid x \in \mathscr{T}_{1}$ int $\left.S(n+1,1 ; y)\right\} \subset \mathscr{T}_{2}$ int $S(n, 2 ; x)$. If we define $\mathscr{L}(m, i)=\{S(x, \mathscr{X}(m, i)) \mid x \in X\}$, then $\mathscr{L}(n+2, i)<\mathscr{X}(n, i)$ for all 
$n=1,2,3 \ldots$ If we write $V(m, i)=\bigcup\left\{\mathscr{T}_{j}\right.$ int $S(m, j ; y) \times \mathscr{T}_{i}$ int $S(m, i ; y) \mid y$ $\in X\}$, then $(x, y) \in V(m+2, i) \circ V(m+2, i)$ implies, for some $z \in X$ that $(x, z) \in V(m+2, i)$ and $(z, y) \in V(m+2, i)$.

Indeed $x \in V(m+2, j)[z] \subset \mathscr{T}_{j}$ int $S(m, j ; z)$ and $y \in V(m+2, i)[z] \subset$ $\mathscr{T}_{i}$ int $S(m, i ; z)$ so that $(x, y) \in V(m, i)$. Also notice that $(V(m, i))^{-1}=V(m, j)$. Thus the conditions are sufficient.

The necessity is proved as follows. Let $p_{1}$ be the quasi-metric that induces $\mathscr{T}_{1}$ and $\mathscr{T}_{2}$ be induced by its conjugate $p_{2}$. Let us write $S(n, i ; x)=\left\{y \mid p_{i}(x, y)<\right.$ $\left.\left(\frac{1}{2}\right)^{n}\right\}$. If $x \notin S(n-1, i ; x)$ and $S(n, i ; x) \cap S(n, j ; z) \neq \varnothing$, then there exists $y \in X$ such that $p_{i}(x, y)<\left(\frac{1}{2}\right)^{n}$ and $p_{j}(z, y)<\left(\frac{1}{2}\right)^{n}$. Hence $p_{i}(x, z) \leqslant p_{i}(x, y)$ $+p_{i}(y, z)<\left(\frac{1}{2}\right)^{n-1}$, a contradiction. Also, if $y \in S(n, i ; x)$ and $z \in S(n, i ; y)$, then $p_{i}(x, y)<\left(\frac{1}{2}\right)^{n}$ and $p_{i}(y, z)<\left(\frac{1}{2}\right)^{n}$ so that $p_{i}(x, z)<\left(\frac{1}{2}\right)^{n-1}$ and hence $z \in S(n-1, i ; x)$.

2. Theorem. A pairwise Hausdorff space $\left(X, \mathscr{T}_{1}, \mathscr{T}_{2}\right)$ is quasi-metrizable if and only if for each $x \in X$ one can assign $\mathscr{T}_{i}$ nbd bases $\{S(n, i ; x) \mid n=1,2, \ldots\}$ such that

(i) $y \notin S(n-1, i ; x)$ implies $S(n, i ; x) \cap S(n, j ; y)=\varnothing$,

(ii) $y \in S(n, i ; x)$ implies $x \in S(n, j ; y)(i, j=1,2 ; i \neq j)$.

Proof. We have to verify only condition (ii) of Theorem 1 . Now

$$
y \notin S(n-1, i ; x)
$$

implies $S(n, i ; x) \cap S(n, j ; y)=\varnothing$ so that if $z \in S(n, i ; x)$, then $z \notin S(n, j ; y)$. Thus $y \notin S(n, i ; z)$. The necessity is obvious.

3. Theorem. A pairwise Hausdorff space $\left(X, \mathscr{T}_{1}, \mathscr{T}_{2}\right)$ is quasi-metrizable if and only if for each $x \in X$ one can assign $\mathscr{T}_{i}$ nbd bases $\{S(n, i ; x) \mid n=1,2, \ldots\}$ such that

(i) $y \in S(n, i ; x)$ implies $S(n, i ; y) \subset S(n-1, i ; x)$,

(ii) $y \in S(n, i ; x)$ implies $x \in S(n, j ; y)(i, j=1,2 ; i \neq j)$.

Proof. We only have to verify condition (i) of Theorem 1 . If

$$
S(n, i ; x) \cap S(n, j ; y) \neq \varnothing,
$$

then there is a point $z \in S(n, i ; x)$ and $z \in S(n, j ; y)$ so that $S(n, i ; z) \subset$ $S(n-1, i ; x)$ and $y \in S(n, i ; z)$. Thus $y \in S(n-1, i ; x)$.

The necessity is obvious.

\section{References}

[1] J. C. Kelly, 'Bitopological spaces', Proc. London Math. Soc. 13 (1963), 71-89.

[2] E. P. Lane, 'Bitopological spaces and quasi-uniform spaces', Proc. London Math. Soc. 17 (1967), 241-256. 
[3] M. C. Murdeshwar and S. A. Naimpally, Quasi-uniform topological spaces (Noordhoff, Groningen, 1966).

[4] C. M. Pareek, 'Bitopological and quasi-metric spaces', J. Univ. Kuwait Sci. 6 (1980), 1-7.

[5] C. W. Patty, 'Bitopological spaces', Duke Math. J. 34 (1967), 387-392.

[6] I. L. Reilly, 'Quasi-guage spaces', J. London Math. Soc. 6 (1973), 481-487.

[7] S. Romoguera, 'Two characterizations of quasi-pseudo-metrizable bitopological spaces', $J$. A ustral. Math. Soc. 35 (1983), 327-333.

[8] S. Romoguera, 'On bitopological quasi-pseudometrization', J. Austral. Math. Soc. 36 (1984), 126-129.

[9] S. Salbany, 'Quasi-metrization of bitopological spaces', Arch. Math. 23 (1972), 299-316.

[10] R. Stoltenberg, 'Some properties of quasi-uniform spaces', Proc. London Math. Soc. 17 (1967), 342-354.

[11] W. A. Wilson, 'On quasi-metric spaces', Amer. J. Math. 53 (1931), 675-684.

\section{Department of Mathematics}

University of Auckland

Private Bag, Auckland

New Zealand 